\title{
Third-Order Conditional Lie-Bäcklund Symmetries of Nonlinear Reaction-Diffusion Equations
}

\author{
Keqin $\mathrm{Su}^{1}$ and Jie $\mathrm{Cao}^{2}$ \\ ${ }^{1}$ College of Information and Management Science, Henan Agricultural University, Zhengzhou 450046, China \\ ${ }^{2}$ College of Information Science and Technology, Donghua University, Shanghai 201620, China \\ Correspondence should be addressed to Keqin Su; keqinsu@hotmail.com
}

Received 24 October 2016; Revised 16 January 2017; Accepted 18 January 2017; Published 9 February 2017

Academic Editor: Nikos Mastorakis

Copyright (c) 2017 Keqin Su and Jie Cao. This is an open access article distributed under the Creative Commons Attribution License, which permits unrestricted use, distribution, and reproduction in any medium, provided the original work is properly cited.

\begin{abstract}
The third-order conditional Lie-Bäcklund symmetries of nonlinear reaction-diffusion equations are constructed due to the method of linear determining equations. As a consequence, the exact solutions of the resulting equations are derived due to the compatibility of the governing equations and the admitted differential constraints, which are resting on the characteristic of the admitted conditional Lie-Bäcklund symmetries to be zero.
\end{abstract}

\section{Introduction}

The conditional Lie-Bäcklund symmetry (CLBS) was independently proposed by Zhdanov [1] and Fokas and Liu [2]. Exact solutions possessing the form of nonlinear separation of variables [3] and exact solutions defined on the invariant subspaces [4] for nonlinear heat conductivity equations were constructed due to the corresponding symmetry reductions in [1], which cannot be obtained within the framework of Lie's classical symmetry [5], conditional symmetry [6, 7], and Lie-Bäcklund symmetry [8]. Multishock and multisoliton solutions of nonintegrable equations were obtained by using CLBS in [2], which are regarded as remnants of integrability for the considered nonintegrable equations.

As a consequence, CLBSs were applied to a number of particular subclasses of evolution equations $[9,10]$. It has been noted that CLBS is very effective in studying classifications and reductions of diffusion equations [11-16]. CLBS can also be used to consider the reductions of initial value problems for nonlinear evolution equations [17-19]. CLBS for evolution systems were studied in [20,21].

The procedure for determining whether or not a given Lie-Bäcklund symmetry is conditionally invariant of the considered equations is straightforward; however, the determination of the most general CLBS admitted by a given differential equation is a very difficult, if not impossible, problem. CLBSs are generalizations of conditional symmetries, in the same way that Lie-Bäcklund symmetries are generalizations of Lie's classical symmetry. Therefore the procedure for computing CLBSs is about the same as that for the conditional symmetries. We need to determine the form of CLBS presumably. It is proved that CLBSs related to separation of variables [13], sign-invariants [14], and invariant subspaces $[15,16]$ are powerful to study different types of diffusion equations. Are there other choices of CLBS which may yield new interesting results for these considered equations?

Once the CLBS and the governing equation are determined, exact solutions of the discussed equations can be easily constructed due to the compatibility of the considered equations and the additional differential constraints obtained by setting the characteristic of the corresponding CLBS to be zero. Thus, CLBS can be reformulated by using the technicalities of the method of differential constraints.

The key idea of the method of differential constraint was proposed by Yanenko in [22]. The general formulation of this method requires that the original system of partial differential equations and the additional differential constraints satisfy some conditions of compatibility. The problem of finding all differential constraints compatible with certain equations can be more complicated than the investigation of solving the original equations. Hence, it is better to content oneself with finding constraints in some fixed classes, and these classes must be chosen using additional considerations. 
In practice, differential constraints related to separation of variables [23] and invariant subspaces [24] are powerful to seek for reductions of different types of evolution equations. Olver and Rosenau [25, 26], Olver [27], Kaptsov [28], and Levi and Winternitz [29] showed that many reductions including Bluman and Cole's nonclassical method [6], partial invariance method [30], Clarkson and Kruskal's direct method [31], and Galaktionov's higher-order direct method [3] can be understood within the framework of differential constraints.

B-determining equations [32] and linear determining equations $[33,34]$ were both proposed to construct differential constraints. The method of linear determining equations with free parameters is much easier to illustrate than the one of B-determining equations with undetermined functions. The linear determining equations are more general than the classical determining equations for Lie's generators [30].

In this paper, we will consider the general third-order CLBS with the characteristic

$$
\eta=u_{3}+g\left(t, x, u, u_{1}, u_{2}\right)
$$

of the nonlinear reaction-diffusion equations

$$
u_{t}=\left(u^{k} u_{x}\right)_{x}+Q(u)
$$

by using the linear determining equations. Equation (2) is widely used as mathematical models of heat conduction and diffusion, filtration of gases and fluids in porous media, process of chemical kinetics, and biological process. It is noted that $u_{j}=\partial^{j} u / \partial x^{j}(j=1,2,3)$ in (1).

There are numerous works aimed to study symmetry classifications and reductions of (2). CLBS was also used to consider the variant forms of (2). Three types of CLBS were applied to study such equations. The CLBS with the characteristic

$$
\eta=[f(u)]_{x t}
$$

was presented to study functionally separable solutions

$$
f(u)=\phi(t)+\psi(x)
$$

of (2) in [13]. The CLBS with the characteristic

$$
\eta=u_{2}+H(u) u_{1}^{2}+G(u) u_{1}+F(u)
$$

was proposed to study the variant forms of (2) in [11, 12], which can give symmetry interpretation for first-order signinvariants of the considered equations. The CLBS with the characteristic

$$
\begin{aligned}
\eta= & {[f(u)]_{n}+a_{1}(x)[f(u)]_{n-1}+a_{2}(x)[f(u)]_{n-2} } \\
& +\cdots+a_{n}(x) f(u)
\end{aligned}
$$

was proposed to study the various forms of $(2)$ in $[15,16]$. The corresponding linearized form

$$
\sigma=u_{n}+a_{1}(x) u_{n-1}+a_{2}(x) u_{n-2}+\cdots+a_{n}(x) u
$$

of (6) is related to the invariant subspace

$$
W=L\left\{f_{1}(x), f_{2}(x), \ldots, f_{n}(x)\right\},
$$

which is the right solution space of the linear ODE $\sigma=0$.

The forms of CLBS in [11-16] are all determined in advance. Thus the corresponding results are of course restricted to this ansatz. The third-order differential constraints (1) of the nonlinear diffusion equation $u_{t}=\left(u^{k} u_{x}\right)_{x}$ are considered in [33]. The differential constraints

$$
\eta=u_{n}+g\left(t, x, u, u_{1}, u_{2}, \ldots, u_{n-1}\right)=0
$$

with $n=2,3$ of (2) are discussed in [34]. However, the study there was worked out for only several special cases. Here we will extend the work there to develop a complete classification for third-order CLBS with the form (1) of (2) by applying the method of linear determining equations. New types of CLBS which are not included in the form of (3), (5), and (6) will be derived and therefore a huge number of new exact solutions of (2) will be constructed due to the compatibility of the governing equations and corresponding resulting differential constraints.

\section{General Statement}

Consider a nonlinear evolution equation

$$
u_{t}=F\left(t, x, u, u_{1}, u_{2}, \ldots, u_{k}\right)
$$

with Lie-Bäcklund vector field (LBVF)

$$
\begin{aligned}
V= & \eta \frac{\partial}{\partial u}+D_{x} \eta \frac{\partial}{\partial u_{1}}+D_{t} \eta \frac{\partial}{\partial u_{t}}+D_{x}^{2} \eta \frac{\partial}{\partial u_{2}} \\
& +D_{x} D_{t} \eta \frac{\partial}{\partial u_{1 t}}+\cdots,
\end{aligned}
$$

where $\eta=\eta\left(x, t, u, u_{1}, u_{t}, u_{t t}, u_{1 t}, \ldots\right)$ is the characteristic of LBVF (11) and the total differentiation operators, respectively, denote

$$
\begin{aligned}
& D_{t}=\frac{\partial}{\partial t}+u_{t} \frac{\partial}{\partial u}+u_{t t} \frac{\partial}{\partial u_{t}}+u_{1 t} \frac{\partial}{\partial u_{1}}+\cdots \\
& D_{x}=\frac{\partial}{\partial x}+u_{1} \frac{\partial}{\partial u}+u_{1 t} \frac{\partial}{\partial u_{t}}+u_{2} \frac{\partial}{\partial u_{1}}+\cdots
\end{aligned}
$$

Definition 1 (see [5]). The evolutionary vector field (11) is said to be a Lie-Bäcklund symmetry of the evolution equation (10) if the condition

$$
\left.V\left(u_{t}-F\right)\right|_{M}=0
$$

holds, where $M$ denotes the set of all differential consequences of (10).

Definition 2 (see $[1,2])$. The evolutionary vector field (11) is said to be a CLBS of (10) if the following condition

$$
\left.V\left(u_{t}-F\right)\right|_{M \cap L_{x}}=0
$$

holds, where $L_{x}$ denotes the set of all differential consequences of equation $\eta=0$ with respect to $x$. 
It is noted that we can exclude all derivatives with respect to $t$ on the set of (10) and thus obtain the vector field (11) with $\eta$ of the form

$$
\eta=\eta\left(t, x, u, u_{1}, u_{2}, \ldots, u_{n}\right)
$$

A direct computation of (14) yields

$$
\left.D_{t} \eta\right|_{M \cap L_{x}}=0
$$

On the other hand, we can derive that

$$
\left.D_{t} \eta\right|_{M \cap L_{x}}=\left.D_{t} \eta\right|_{M_{x} \cap L_{x}}
$$

where $M_{x}$ denotes the set of all differential consequences of (10) with respect to $x$. Thus, we have that the condition

$$
\left.D_{t} \eta\right|_{M_{x} \cap L_{x}}=0
$$

holds, which is exactly the sufficient condition $[28,35]$ for which $\eta=0$ is a differential constraint of the evolution equation (10). Thus $\eta=0$ defines an invariant manifold of the equation (10), which is right corresponding to the symmetry reductions due to the compatibility of $\eta=0$ and the considered (10). There is an equivalence relation between CLBS and differential constraint for the evolution equation (10).

Kaptsov [33, 34] proposed a method for finding differential constraints, which uses linear determining equations. The definition of linear determining equation was first given in [33], which is listed as follows.

Definition 3 (see $[33,34]$ ). A linear determining equation corresponding to differential constraints $\eta\left(t, x, u, u_{1}, u_{2}, \ldots\right.$, $\left.u_{n}\right)=0$ of the evolution equation (10) is an equation of the form

$$
\left.D_{t} \eta\right|_{M_{x}}=\sum_{i=0}^{n} \sum_{j=0}^{i} b_{i j} D_{x}^{i-j}\left(F_{u_{n-j}}\right) D_{x}^{n-i}(\eta),
$$

where $b_{i j}$ are some constants.

\section{CLBS of (2)}

As stated in [34], the linear determining equation for the differential constraints $\eta=0$ of (2) is right

$$
\begin{aligned}
\left.D_{t} \eta\right|_{E_{x}} & \\
= & u^{k} D_{x}^{2} \eta+b_{1} k u^{k-1} u_{1} D_{x} \eta \\
& +\left[b_{3} k u^{k-1} u_{2}+b_{2} k(k-1) u^{k-2} u_{1}^{2}+b_{4} f_{u}\right] \eta,
\end{aligned}
$$

where $b_{1}, b_{2}, b_{3}, b_{4} \in \mathbb{R}$ and $E_{x}$ is the differential consequences of (2) with respect to $x$. It is easy to see that (2) admits LBVF (11) if the condition (20) holds.
Substituting (1) into (20), the left-hand side and the righthand side are, respectively, simplified as

$$
\begin{aligned}
& \left.D_{t} \eta\right|_{E_{x}}=u^{k} u_{5}+u^{k-1}\left(u g_{u_{2}}+5 k u_{1}\right) u_{4}+\left[u^{k} g_{u_{1}}\right. \\
& +4 k u^{k-1} u_{1} g_{u_{2}}+10 k u^{k-1} u_{2}+10 k(k-1) u^{k-2} u_{1}^{2} \\
& \left.+Q_{u}\right] u_{3}+\left[k(k-1)(k-2) u^{k-3} u_{1}^{4}+3 k u^{k-1} u_{2}^{2}\right. \\
& \left.+6 k(k-1) u^{k-2} u_{2} u_{1}^{2}+u_{1}^{2} Q_{u u}+u_{2} Q_{u}\right] g_{u_{2}} \\
& +\left[u_{1} Q_{u}+k(k-1) u^{k-2} u_{1}^{3}+3 k u^{k-1} u_{2} u_{1}\right] g_{u_{1}} \\
& +\left(u^{k} u_{2}+k u^{k-1} u_{1}^{2}+Q_{u}\right) g_{u}+g_{t}+u_{1}^{3} Q_{u u u} \\
& +3 u_{2} u_{1} Q_{u u}+k(k-1)(k-2)(k-3) u^{k-4} u_{1}^{5} \\
& +10 k(k-1)(k-2) u^{k-3} u_{2} u_{1}^{3}+15 k(k-1) \\
& \cdot u^{k-2} u_{2}^{2} u_{1} \\
& u^{k} D_{x}^{2} \eta+b_{1} k u^{k-1} u_{1} D_{x} \eta+\left[b_{3} k u^{k-1} u_{2}\right. \\
& \left.+b_{2} k(k-1) u^{k-2} u_{1}^{2}+b_{4} f_{u}\right] \eta=u^{k} u_{5}+u^{k-1}\left(u g_{u_{2}}\right. \\
& \left.+b_{1} k u_{1}\right) u_{4}+u^{k} g_{u_{2} u_{2}} u_{3}^{2}+\left[b_{4} Q_{u}\right. \\
& +u^{k}\left(2 u_{1} g_{u u_{2}}+2 g_{x u_{2}}+2 u_{2} g_{u_{1} u_{2}}+g_{u_{1}}\right) \\
& \left.+b_{1} k u^{k-1} u_{1} g_{u_{2}}+b_{2} k(k-1) u^{k-2} u_{1}^{2}+b_{3} k u^{k-1} u_{2}\right] \\
& \cdot u_{3}+u^{k}\left(u_{2}^{2} g_{u_{1} u_{1}}+2 u_{1} u_{2} g_{u u_{1}}+u_{1}^{2} g_{u u}+2 u_{2} g_{x u_{1}}\right. \\
& \left.+2 u_{1} g_{x u}+g_{x x}+u_{2} g_{u}\right)+b_{1} k u^{k-1} u_{1}\left(u_{2} g_{u_{1}}\right. \\
& \left.+u_{1} g_{u}+g_{x}\right)+\left[b_{3} k u^{k-1} u_{2}+b_{2} k(k-1) u^{k-2} u_{1}^{2}\right. \\
& \left.+b_{4} Q_{u}\right] g
\end{aligned}
$$

which can be regarded as two polynomials with respect to $u_{5}, u_{4}$, and $u_{3}$. It is noted that the subscripts here denote the differentiation with respect to the indicated variables.

Considering the coefficients of $u_{4}$ and $u_{3}^{2}$, we obtain

$$
\begin{aligned}
b_{1} & =5, \\
g_{u_{2} u_{2}} & =0 .
\end{aligned}
$$

So $g$ can be represented by

$$
g=h\left(t, x, u, u_{1}\right) u_{2}+f\left(t, x, u, u_{1}\right) .
$$

Here $h$ and $f$ are both functions of $t, x, u$, and $u_{1}$. Collecting the coefficients of $u_{3}$, we have

$$
\begin{aligned}
& {\left[2 u^{k} h_{u_{1}}+\left(b_{3}-10\right) k u^{k-1}\right] u_{2}+2 u^{k}\left(u_{1} h_{u}+h_{x}\right)} \\
& \quad+k u^{k-1} u_{1} h+k(k-1)\left(b_{2}-10\right) u^{k-2} u_{1}^{2} \\
& \quad+\left(b_{4}-1\right) Q_{u}=0 .
\end{aligned}
$$


Thus, we know that $h$ must satisfy

$$
\begin{aligned}
& 2 u^{k} h_{u_{1}}+\left(b_{3}-10\right) k u^{k-1}=0, \\
& 2 u^{k}\left(u_{1} h_{u}+h_{x}\right)+k u^{k-1} u_{1} h \\
& \quad+k(k-1)\left(b_{2}-10\right) u^{k-2} u_{1}^{2}+\left(b_{4}-1\right) Q_{u}=0 .
\end{aligned}
$$

Solving (25), we obtain

$$
h=-\frac{k\left(b_{3}-10\right)}{2 u} u_{1}+a(t, x, u)
$$

and $a(t, x, u)$ is the function of $t, x$, and $u$. As a consequence, (26) can be simplified as

$$
\begin{aligned}
& k\left(5 k-k b_{2}+\frac{1}{2} k b_{3}+b_{2}-b_{3}\right) u^{k-2} u_{1}^{2} \\
& \quad-\left(2 u a_{u}+k u^{k-1} a\right) u_{1}-2 u a_{x}+\left(1-b_{4}\right) Q_{u}=0,
\end{aligned}
$$

which implies

$$
\begin{aligned}
k\left(5 k-k b_{2}+\frac{1}{2} k b_{3}+b_{2}-b_{3}\right) & =0, \\
2 u a_{u}+k u^{k-1} a & =0, \\
-2 u a_{x}+\left(1-b_{4}\right) Q_{u} & =0 .
\end{aligned}
$$

Solving the linear ordinary differential equation about $a$, we get $a=C(t, x) u^{-k / 2}$. The simplification of the above equation about $Q$ gives

$$
\left(1-b_{4}\right) Q_{u}-2 u^{k / 2} C_{x}=0 .
$$

We know that $C_{x}=d_{1}$ must hold and then the above equations about $Q$ become

$$
\left(1-b_{4}\right) Q_{u}-2 d_{1} u^{k / 2}=0 .
$$

Solving this ordinary differential equation, we know that there are three subcases for further discussion, which are listed as

$$
\begin{aligned}
& b_{4}=1 \\
& d_{1}=0
\end{aligned}
$$

(2)

$$
\begin{aligned}
& k=-2, \\
& Q=\frac{2 d_{1} \ln (u)}{1-b_{4}}+d_{3} ;
\end{aligned}
$$

(3)

$$
Q=\frac{2 d_{1} u^{1+k / 2}}{\left(1-b_{4}\right)(1+k / 2)}+d_{3}, \quad b_{4} \neq 1, k \neq-2 .
$$

For these three different cases, a further identical vanishing of (20) will finally determine the form of $g$ in (1). The detailed procedure is omitted here. We just present the corresponding results in Table 1 , where $d_{i}(i=1,2,3,4,5)$ are arbitrary constants.

\section{Exact Solutions of (2)}

In this section, we construct exact solutions of (2) due to the compatibility of differential constraints $\eta=0$ and the governing equation. The equivalence relation between CLBSs and differential constraints for the evolution equation (10) is first rigorously proved in [36], where it is also stated that finding of the whole set of CLBSs of an evolution equation is a no-go problem. In fact, the general forms CLBSs $\eta$ of (2) are completely determined by solving the linear system of determining equations about $\eta$, which are listed in Table 1. Solving $\eta=0$, we can obtain $u$ as a function of $x$ with $t$ dependent undetermined functions. Substituting the form of $u$ into (2) will yield that the $t$-dependent coefficients in (2) satisfy three-dimensional dynamical system. Here we give several examples to illustrate the reduction procedure.

Example 1. Equation

$$
u_{t}=\left(u^{k} u_{x}\right)_{x}+d_{1} u+d_{2} u^{1-k}+\frac{(k+1) d_{3}}{k^{2}} u^{1+k}
$$

admits the CLBS

$$
\eta=u_{3}+\frac{3(k-1)}{u} u_{1} u_{2}+\frac{(k-1)(k-2)}{u^{2}} u_{1}^{3}+d_{3} u_{1} .
$$

The corresponding solutions are given as follows.

(i) For $d_{3}>0$,

$$
\begin{aligned}
& u(x, t) \\
& =\left[\alpha(t)+\beta(t) \sin \left(\sqrt{d_{3}} x\right)+\gamma(t) \cos \left(\sqrt{d_{3}} x\right)\right]^{1 / k},
\end{aligned}
$$

where $\alpha(t), \beta(t)$, and $\gamma(t)$ satisfy three-dimensional dynamical system

$$
\begin{aligned}
& \alpha^{\prime}=\frac{(k+1) d_{3}}{k} \alpha^{2}+\frac{d_{3}}{k} \beta^{2}+\frac{d_{3}}{k} \gamma^{2}+k d_{1} \alpha+k d_{2}, \\
& \beta^{\prime}=\frac{(k+2) d_{3}}{k} \alpha \beta+k d_{1} \beta, \\
& \gamma^{\prime}=\frac{(k+2) d_{3}}{k} \alpha \gamma+k d_{1} \gamma .
\end{aligned}
$$

(ii) For $d_{3}<0$,

$$
\begin{aligned}
& u(x, t)=\left[\alpha(t)+\beta(t) \sinh \left(\sqrt{-d_{3}} x\right)\right. \\
& \left.\quad+\gamma(t) \cosh \left(\sqrt{-d_{3}} x\right)\right]^{1 / k}
\end{aligned}
$$

where $\alpha(t), \beta(t)$, and $\gamma(t)$ satisfy three-dimensional dynamical system

$$
\begin{aligned}
& \alpha^{\prime}=\frac{(k+1) d_{3}}{k} \alpha^{2}-\frac{d_{3}}{k} \beta^{2}+\frac{d_{3}}{k} \gamma^{2}+k d_{1} \alpha+k d_{2}, \\
& \beta^{\prime}=\frac{(k+2) d_{3}}{k} \alpha \beta+k d_{1} \beta, \\
& \gamma^{\prime}=\frac{(k+2) d_{3}}{k} \alpha \gamma+k d_{1} \gamma .
\end{aligned}
$$




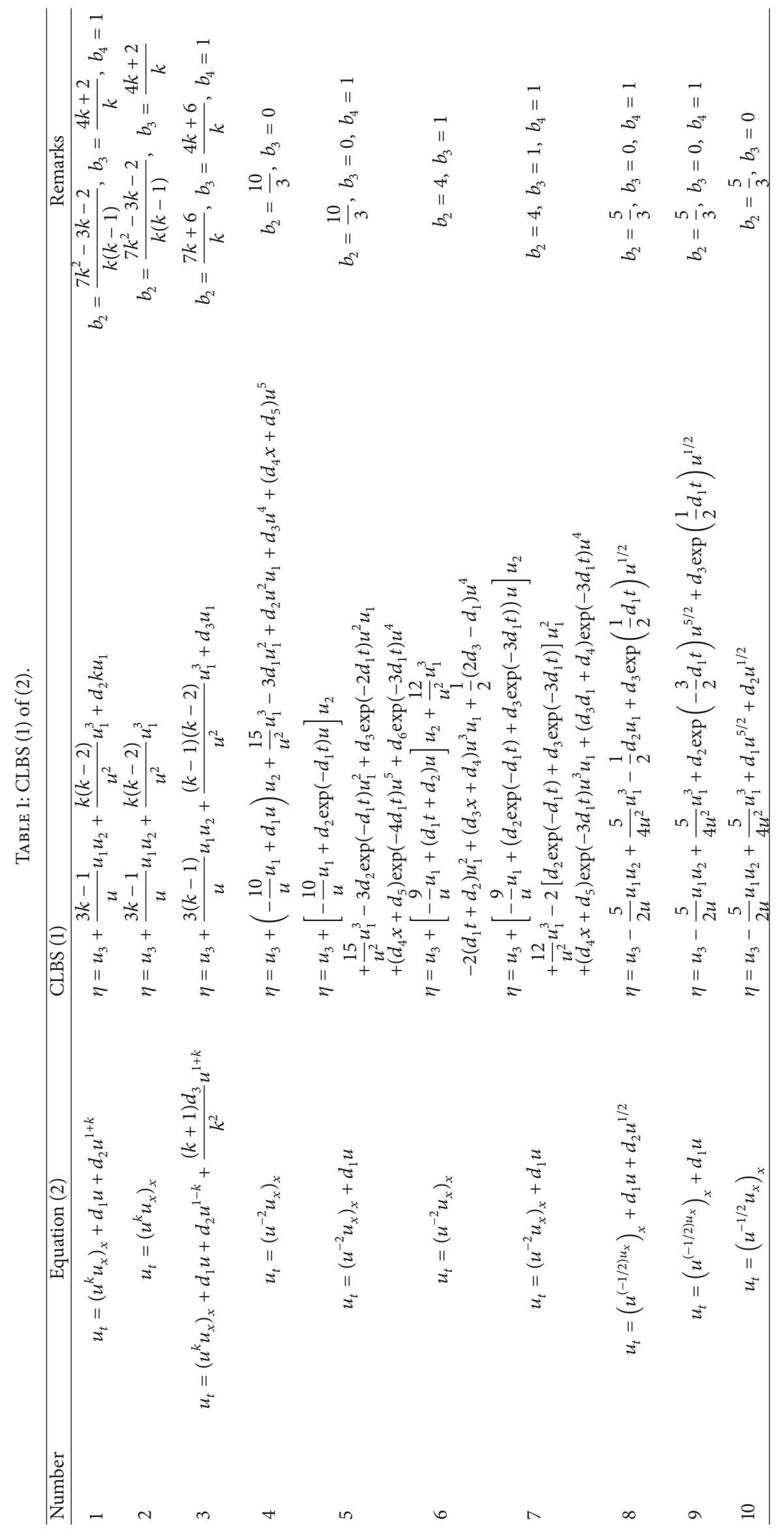




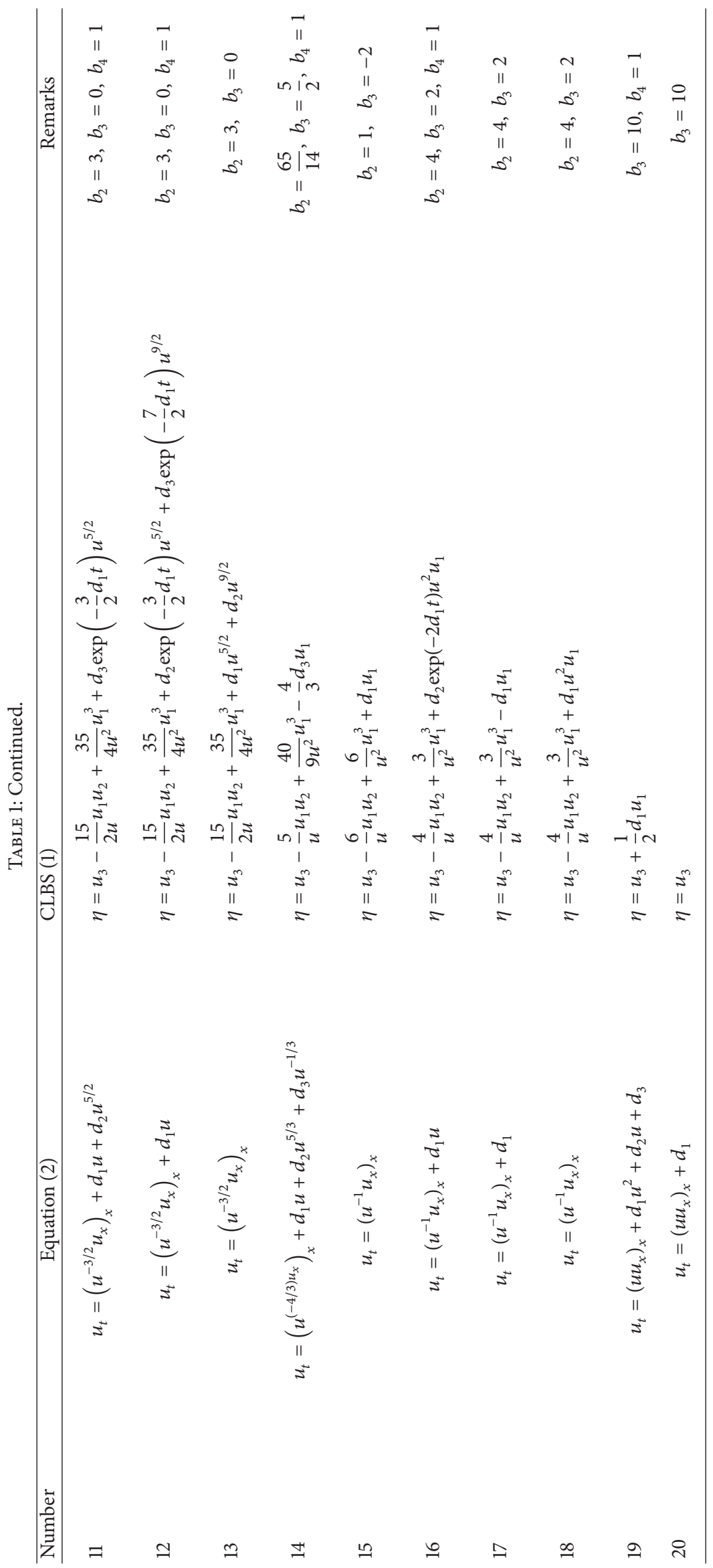


(iii) For $d_{3}=0$,

$$
u(x, t)=\left[\alpha(t)+\beta(t) x+\gamma(t) x^{2}\right]^{1 / k},
$$

where $\alpha(t), \beta(t)$, and $\gamma(t)$ satisfy three-dimensional dynamical system

$$
\begin{aligned}
& \alpha^{\prime}=\frac{1}{k} \beta^{2}+2 \alpha \gamma+k d_{1} \alpha+k d_{2}, \\
& \beta^{\prime}=\frac{2(k+2)}{k} \beta \gamma+k d_{1} \beta, \\
& \gamma^{\prime}=\frac{2(k+2)}{k} \gamma^{2}+k d_{1} \gamma .
\end{aligned}
$$

Example 2. Equation

$$
u_{t}=\left(u^{-4 / 3} u_{x}\right)_{x}+d_{1} u+d_{2} u^{5 / 3}
$$

admits the CLBS

$$
\eta=u_{3}-\frac{5}{u} u_{1} u_{2}+\frac{40}{9 u^{2}} u_{1}^{3} .
$$

The corresponding solutions of (43) are given by

$$
u(x, t)=\left[\alpha(t)+\beta(t) x+\gamma(t) x^{2}\right]^{-3 / 2},
$$

where $\alpha(t), \beta(t)$, and $\gamma(t)$ satisfy three-dimensional dynamical system

$$
\begin{aligned}
& \alpha^{\prime}=2 \alpha^{2} \gamma-\frac{1}{2} \alpha \beta^{2}-\frac{2}{3} d_{1} \alpha-\frac{2}{3} d_{2}, \\
& \beta^{\prime}=-\frac{1}{2} \beta^{3}+2 \alpha \beta \gamma-\frac{2}{3} d_{1} \beta, \\
& \gamma^{\prime}=2 \alpha \gamma^{2}-\frac{1}{2} \beta^{2} \gamma-\frac{2}{3} d_{1} \gamma .
\end{aligned}
$$

Example 3. Equation

$$
u_{t}=\left(u^{-1} u_{x}\right)_{x}+d_{1} u^{2}+d_{2} u
$$

admits the CLBS

$$
\eta=u_{3}-\frac{6}{u} u_{1} u_{2}+\frac{6}{u^{2}} u_{1}^{3} .
$$

The corresponding solutions are given by

$$
u(x, t)=-\frac{\beta(t)}{\alpha(t)-\left(x+c_{1}\right)^{2}},
$$

where $\alpha(t), \beta(t)$, and $\gamma(t)$ are listed as follows.

(i) For $d_{2} \neq 0$,

$$
\begin{aligned}
& \alpha(t)=\left[\frac{2 d_{1} \ln \left(c_{2} d_{2} \exp \left(d_{2} t\right)-2\right)}{d_{2}^{4} c_{2}^{2}}+\frac{d_{1} \exp \left(d_{2} t\right)}{d_{2}^{3} c_{2}}\right. \\
& \left.+c_{3}\right]\left[c_{2} d_{2}-2 \exp \left(-d_{2} t\right)\right],
\end{aligned}
$$

$$
\beta(t)=c_{2} \exp \left(d_{1} t\right)-\frac{2}{d_{2}} \text {. }
$$

(ii) For $d_{2}=0$,

$$
\begin{aligned}
& \alpha(t)=\left[\frac{1}{2} d_{1} \ln \left(2 t+c_{2}\right)+c_{3}\right]\left(2 t+c_{2}\right)^{2}, \\
& \beta(t)=2 t+c_{2} .
\end{aligned}
$$

Example 4. Equation

$$
u_{t}=\left(u^{-1} u_{x}\right)_{x}
$$

admits the CLBS

$$
\eta=u_{3}-\frac{4}{u} u_{1} u_{2}+\frac{3}{u^{2}} u_{1}^{3}+d_{1} u^{2} u_{1} .
$$

The corresponding solutions are listed as follows.

(i) For $d_{1}>0$,

$$
u(x, t)=\frac{-4 d_{1} c_{1}^{2} \exp \left[-\sqrt{c_{1}^{2} / d_{1}}(x+\gamma(t))\right]}{-\exp \left[-2 \sqrt{c_{1}^{2} / d_{1}}(x+\gamma(t))\right]+4 d_{1}^{3} \exp \left[-\sqrt{c_{1}^{2} / d_{1}}(x+\gamma(t))\right] \beta(t)-4 d_{1}^{4} \alpha(t)},
$$

where the unknown functions about $t$ are, respectively, given as

$$
\begin{aligned}
& \alpha(t)=c_{1}^{2}\left[1+\tan ^{2}\left(c_{1} t+c_{2}\right)\right], \\
& \beta(t)=-\frac{c_{1}}{d_{1}} \tan \left(c_{1} t+c_{2}\right),
\end{aligned}
$$

(ii) For $d_{1}<0$, 


$$
u(x, t)=\frac{4 d_{1} c_{1}^{2} \exp \left[-\sqrt{-c_{1}^{2} / d_{1}}(x+\gamma(t))\right]}{-\exp \left[-2 \sqrt{-c_{1}^{2} / d_{1}}(x+\gamma(t))\right]+4 d_{1}^{3} \exp \left[-\sqrt{-c_{1}^{2} / d_{1}}(x+\gamma(t))\right] \beta(t)-4 d_{1}^{4} \alpha(t)} .
$$

The unknown functions in the solutions are, respectively, given as

$$
\begin{aligned}
& \alpha(t)=c_{1}^{2}\left[\tanh ^{2}\left(c_{1} t+c_{2}\right)-1\right], \\
& \beta(t)=\frac{c_{1}}{d_{1}} \tanh \left(c_{1} t+c_{2}\right), \\
& \gamma(t)=c_{3}+\frac{1}{2} \sqrt{-\frac{d_{1}}{c_{1}^{2}}} \ln \left[\tanh ^{2}\left(c_{1} t+c_{2}\right)-1\right] .
\end{aligned}
$$

Exact solutions of the fast diffusion equation (52) are investigated from the symmetry point of view in many literatures. The widest and most arranged collection of such solutions is given in [37]. The second-order CLBS and corresponding symmetry reductions for (52) are referred to [12]. The solutions constructed here are not equivalent to the ones in $[12,37]$.

\section{Conclusions}

In this paper, the linear determining equations with parameters are applied to construct CLBSs of the nonlinear reactiondiffusion equation (2). The method generalizes the determining equations used in the search for admissible Lie operators. The procedure for the derivation of exact solutions to the evolution equation (10) is sketched as below. Firstly, finding solutions of the linear determining equations (20), we will obtain the explicit form of CLBSs (1) for (2). Solving the obtained differential constraints, we obtain $u$ including three arbitrary functions depending on $t$. Consequently, substituting the form of $u$ into (10) will lead to three-dimensional dynamical system for the undetermined functions about $t$.

We are mainly concerned with third-order CLBSs of (2) in the present paper. The corresponding exact solutions are constructed due to the resulting symmetry reductions. Naturally, a question will arise: how complicated can the CLBS admitted by the evolution equation (10) be? In other words, what is the vast value of $n$ in the CLBS (9) of (10)? For linear case (7), the question is answered by Svirshchevskii in [38] due to the dimension estimate of invariant subspace admitted by the nonlinear operator $F$, where $F[u]=F\left(x, t, u, u_{1}, \ldots, u_{k}\right)$. Does the resulting relation $n \leq 2 k+1$ there is still effective for the nonlinear case? This will be involved in our future study.

\section{Competing Interests}

The authors declare that there is no conflict of interests regarding the publication of this paper.

\section{Acknowledgments}

This work was supported by Key Scientific and Technological Project of Higher Education of Henan Province (no. 16A110032).

\section{References}

[1] R. Z. Zhdanov, "Conditional Lie-Backlund symmetry and reduction of evolution equations," Journal of Physics A: Mathematical and General, vol. 28, no. 13, pp. 3841-3850, 1995.

[2] A. S. Fokas and Q. M. Liu, "Nonlinear interaction of traveling waves of nonintegrable equations," Physical Review Letters, vol. 72, no. 21, pp. 3293-3296, 1994.

[3] V. A. Galaktionov and V. M. Matrosov, "On new exact blow-up solutions for nonlinear heat conduction equations with source and applications," Differential and Integral Equations, vol. 3, no. 5, pp. 863-874, 1990.

[4] V. A. Galaktionov, "Invariant subspaces and new explicit solutions to evolution equations with quadratic nonlinearities," Proceedings of the Royal Society of Edinburgh: Section A Mathematics, vol. 125, no. 2, pp. 225-246, 1995.

[5] G. W. Bluman and S. Kumei, Symmetries and Differential Equations, Springer, New York, NY, USA, 1989.

[6] G. W. Bluman and J. D. Cole, "The general similarity solution of the heat equation," Journal of Applied Mathematics and Mechanics, vol. 18, pp. 1025-1042, 1996.

[7] W. I. Fushchych, W. M. Shtelen, and N. I. Serov, Symmetry Analysis and Exact Solutions of Nonlinear Equations of Mathematical Physics, Naukova Dumka, Kiev, Ukraine, 1989, Translated into English by Kluwer Academic Publishers, Dordrecht, The Netherlands, 1993.

[8] N. K. Ibragimov, Transformation Groups Applied to Mathematical Physics, Reidel, Boston, Mass, USA, 1985.

[9] Q. M. Liu, "Exact interaction of solitary waves for certain nonintegrable equations," Journal of Mathematical Physics, vol. 37, no. 1, pp. 324-345, 1996.

[10] Q. M. Liu, "Exact solutions to nonlinear equations with quadratic nonlinearity," Journal of Physics A: Mathematical and General, vol. 34, no. 24, pp. 5083-5088, 2001.

[11] C. Z. Qu, "Group classification and generalized conditional symmetry reduction of the nonlinear diffusion-convection equation with a nonlinear source," Studies in Applied Mathematics, vol. 99, no. 2, pp. 107-136, 1997.

[12] C. Z. Qu, "Exact solutions to nonlinear diffusion equations obtained by a generalized conditional symmetry," IMA Journal of Applied Mathematics, vol. 62, no. 3, pp. 283-302, 1999.

[13] C. Qu, S. Zhang, and R. Liu, "Separation of variables and exact solutions to quasilinear diffusion equations with nonlinear source," Physica D: Nonlinear Phenomena, vol. 144, no. 1-2, pp. 97-123, 2000. 
[14] C. Z. Qu, L. N. Ji, and L. Z. Wang, "Conditional lie Bäcklund symmetries and sign-invariants to quasi-linear diffusion equations," Studies in Applied Mathematics, vol. 119, no. 4, pp. 355391, 2007.

[15] L. Ji and C. Qu, "Conditional Lie-Bäcklund symmetries and invariant subspaces to non-linear diffusion equations," IMA Journal of Applied Mathematics (Institute of Mathematics and Its Applications), vol. 76, no. 4, pp. 610-632, 2011.

[16] L. Ji and C. Qu, "Conditional Lie-Bäcklund symmetries and invariant subspaces to nonlinear diffusion equations with convection and source," Studies in Applied Mathematics, vol. 131, no. 3, pp. 266-301, 2013.

[17] P. Basarab-Horwath and R. Z. Zhdanov, "Initial-value problems for evolutionary partial differential equations and higher-order conditional symmetries," Journal of Mathematical Physics, vol. 42, no. 1, pp. 376-389, 2001.

[18] R. Z. Zhdanov and A. Y. Andreitsev, "Non-classical reductions of initial-value problems for a class of nonlinear evolution equations," Journal of Physics A: Mathematical and General, vol. 33, no. 32, pp. 5763-5781, 2000.

[19] R. Z. Zhdanov, "Higher conditional symmetry and reduction of initial value problems," Nonlinear Dynamics, vol. 28, no. 1, pp. $17-27,2002$.

[20] A. Sergyeyev, "Constructing conditionally integrable evolution systems in $(1+1)$ dimensions: a generalization of invariant modules approach," Journal of Physics A: Mathematical and General, vol. 35, no. 35, pp. 7653-7660, 2002.

[21] L. N. Ji, C. Z. Qu, and S. Shen, "Conditional Lie-Bäcklund symmetry of evolution system and application for reactiondiffusion system," Studies in Applied Mathematics, vol. 133, no. 1, pp. 118-149, 2014.

[22] N. N. Yanenko, "Theory of consistency and methods of integrating systems of nonlinear partial differential equations," in Proceedings of the 4th All-Union Mathematical Congress, pp. 247-259, Leningrad, Russia, 1964 (Russian).

[23] E. Pucci and G. Saccomandi, "Evolution equations, invariant surface conditions and functional separation of variables," Physica D, vol. 139, no. 1-2, pp. 28-47, 2000.

[24] V. A. Galaktionov and S. Svirshchevski, Exact Solutions and Invariant Subspaces of Nonlinear Partial Differential Equations in Mechanics and Physics, Chapman \& Hall, London, UK, 2007.

[25] P. J. Olver and P. Rosenau, "The construction of special solutions to partial differential equations," Physics Letters A, vol. 114, no. 3, pp. 107-112, 1986.

[26] P. J. Olver and P. Rosenau, "Group invariant solutions of differential equations," SIAM Journal on Applied Mathematics, vol. 47, no. 2, pp. 263-278, 1987.

[27] P. J. Olver, "Direct reduction and differential constraints," Proceedings of the Royal Society of London, Series A: Mathematical and Physical Sciences, vol. 444, no. 1922, pp. 509-523, 1994.

[28] O. V. Kaptsov, "Invariant sets of evolution equations," Nonlinear Analysis: Theory, Methods \& Applications, vol. 19, no. 8, pp. 753761, 1992.

[29] D. Levi and P. Winternitz, "Non-classical symmetry reduction: example of the boussinesq equation," Journal of Physics A, vol. 22, no. 15 , pp. 2915-2924, 1989.

[30] L. V. Ovsiannikov, Group Analysis of Differential Equations, Academic Press, New York, NY, USA, 1982.

[31] P. A. Clarkson and M. D. Kruskal, "New similarity reductions of the Boussinesq equation," Journal of Mathematical Physics, vol. 30, no. 10, pp. 2201-2213, 1989.
[32] O. V. Kaptsov, "B-determining equations: applications to nonlinear partial differential equations," European Journal of Applied Mathematics, vol. 6, no. 3, pp. 265-286, 1995.

[33] O. V. Kaptsov, "Linear determining equations for differential constraints," Sbornik: Mathematics, vol. 189, no. 11-12, pp. 1839$1854,1998$.

[34] O. V. Kaptsov and I. V. Verevkin, "Differential constraints and exact solutions of nonlinear diffusion equations," Journal of Physics A: Mathematical and General, vol. 36, no. 5, pp. 14011414, 2003.

[35] V. K. Andreev, O. V. Kaptsov, V. V. Pukhnachov, and A. A. Rodionov, Applications of Group-Theoretical Methods in Hydrodynamics, Springer, Dordrecht, Netherlands, 1998.

[36] M. Kunzinger and R. O. Popovych, "Generalized conditional symmetries of evolution equations," Journal of Mathematical Analysis and Applications, vol. 379, no. 1, pp. 444-460, 2011.

[37] R. O. Popovych, O. O. Vaneeva, and N. M. Ivanova, "Potential nonclassical symmetries and solutions of fast diffusion equation," Physics Letters A, vol. 362, no. 2-3, pp. 166-173, 2007.

[38] S. R. Svirshchevskii, "Nonlinear differential operators of first and second order possessing invariant linear spaces of maximal dimension," Theoretical and Mathematical Physics, vol. 105, no. 2, pp. 1346-1353, 1995. 


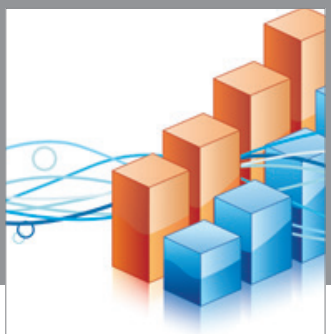

Advances in

Operations Research

vatem alat4

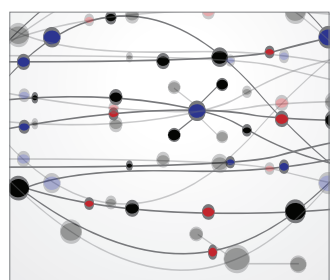

\section{The Scientific} World Journal
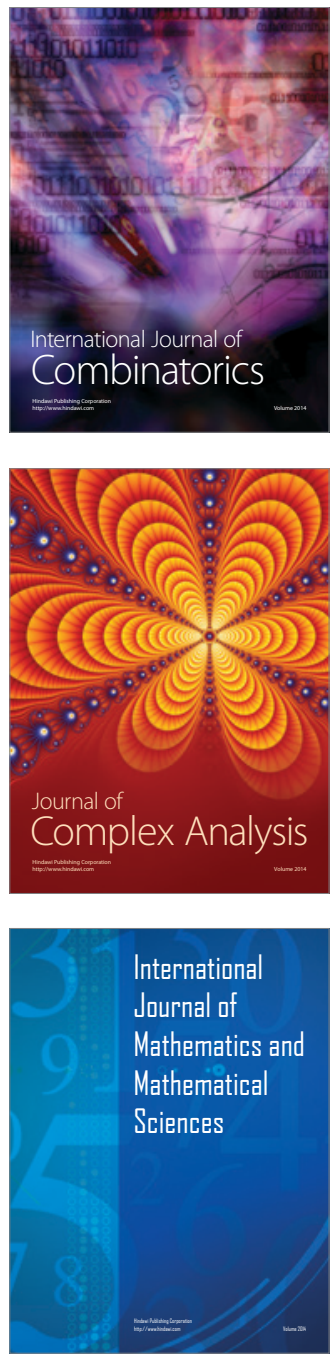
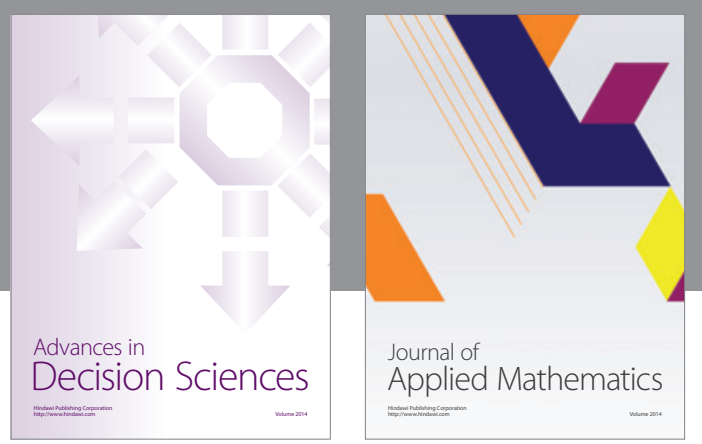

Algebra

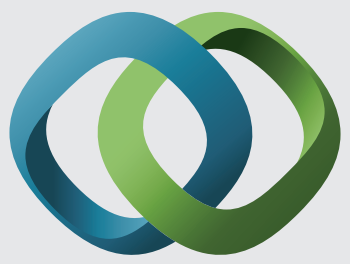

\section{Hindawi}

Submit your manuscripts at

https://www.hindawi.com
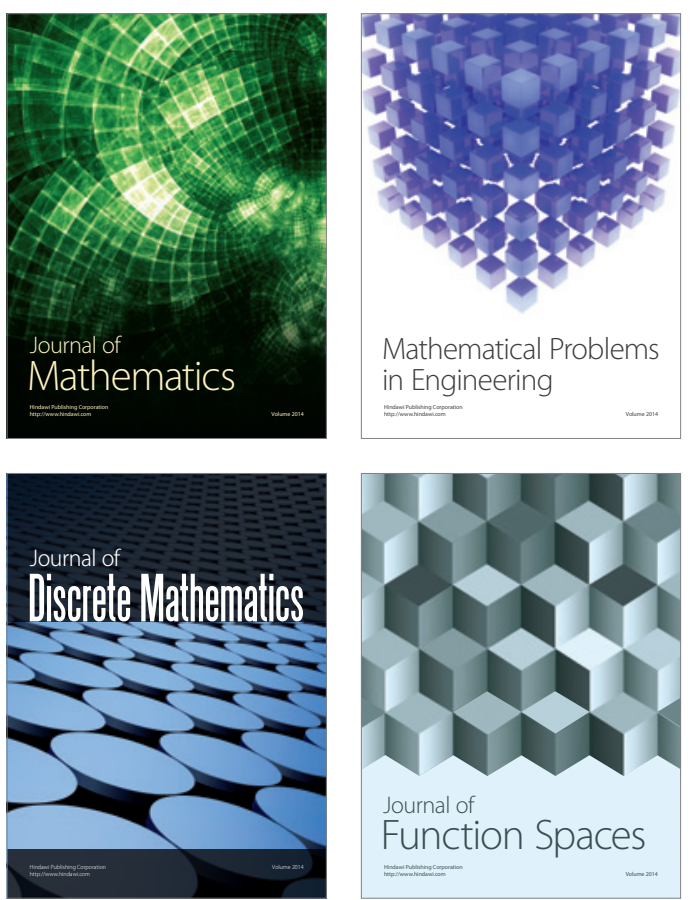

Mathematical Problems in Engineering
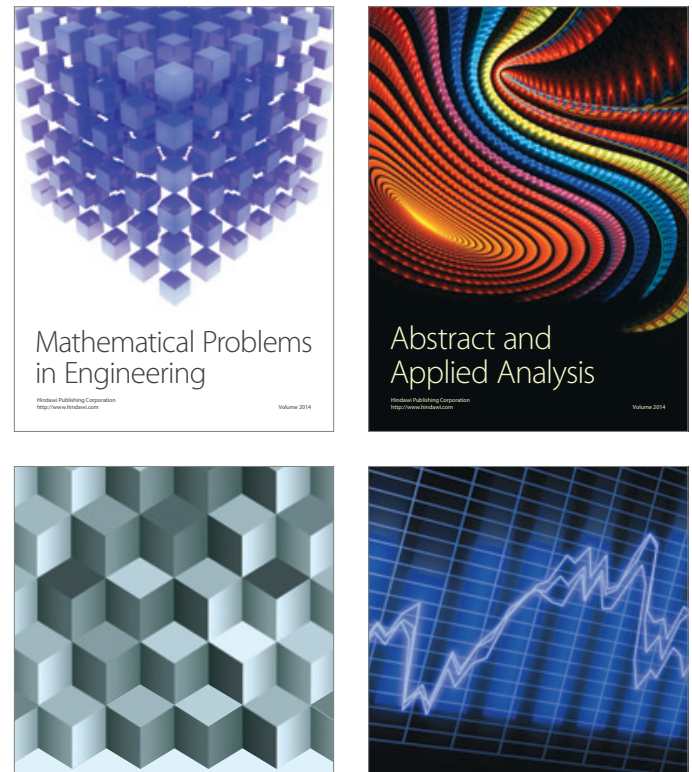

Journal of

Function Spaces

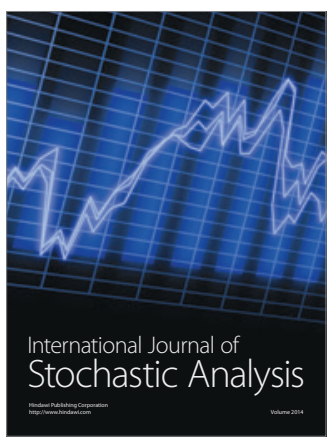

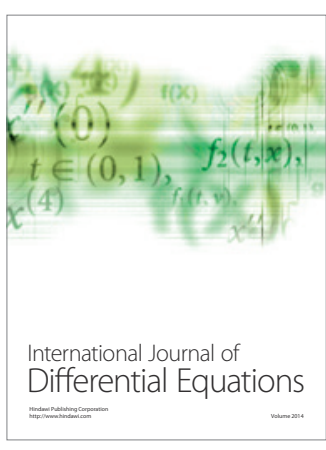
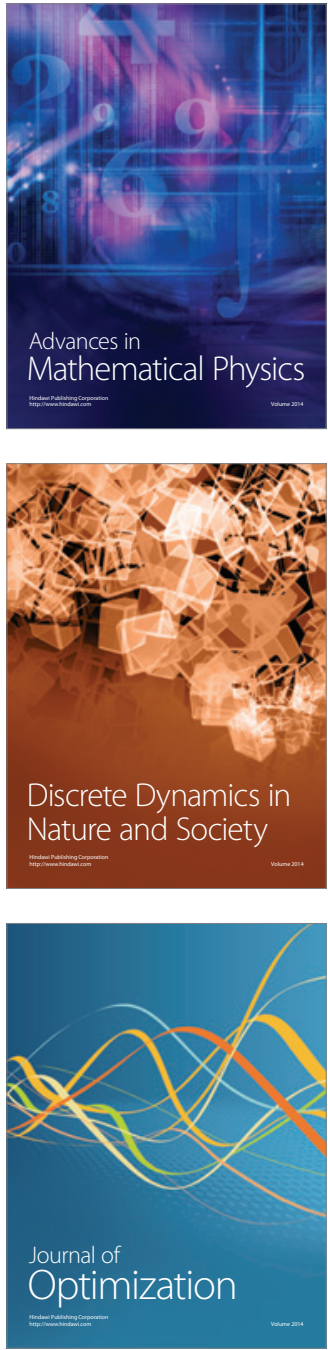